\title{
Design and Evaluation of Multi-storey Lifting Frame Bridge Based Automated Container Terminal Handling System
}

\author{
Ting Fang, Wei Yan, Youfang Huang, Junliang He and Danping Lin \\ Engineering Research Center of Container Supply Chain Technology, Ministry of \\ Education, Shanghai Maritime University, Shanghai 201306, P. R. China \\ fangting_china@126.com
}

\begin{abstract}
This paper aims to propose an innovated design of automated container terminal (ACT) handling system, which can help the port to enhance the handling performance of the entire terminal. Different from conventional ACTs that use trucks or automated guided vehicles $(A G V s)$ to transport containers in the terminal, the new handling system takes advantages of multi-storey lifting frame bridge (MLFB), elevators, automated lifting vehicles (ALVs), frame trolleys (FTs) and reach stackers(RSs). The MLFB-based ACT handling system is composed of several subsystems, which have respective lifting frame bridges. Furthermore, an explorative study has been applied to analyze the transportation efficiency within the handling system using a queuing theory model. The results represent significant improvements over that of conventional handling systems.
\end{abstract}

Keywords: automated container terminal, multi-storey lifting frame bridge, elevator, automated lifting vehicle, evaluation

\section{Introduction}

Nowadays, port operators face a complex, continuously changing and uncertain environment through trends and changes in the area of globalization. Technological changes and innovations are occurred in the world container industry, especially with the increasing number of containers and the advent of mega-vessels. To cope with such uncertain and changing environment, port operators strive for new innovation to handle these containers. At the same time, it is difficult for terminal to expand its capacity fast enough due to the scarce land space, availability of initial investment and environmental concerns (Le-Griffin et al., 2006). How to effectively improve the handling efficiency of terminal and reduce operating cost has greatly attracted the attention of the port operators and researchers. Given these reasons, port operators and researches try to find alternatives which can handle efficiently and lower the cost at the same time. As a result, the concept of automated container terminal (ACT) has become very popular among port operators as a way to improve efficiency, reduce cost, and increase capacity. ACTs are potential candidates for improving the performance of container terminals and meeting the challenges of the future in marine transportation. Recent advances in electronics, sensors, information technologies and automation make the development of fully automated terminals technically feasible.

In the modern ACTs, port operators use different kinds of equipments to handle the container flow. Take European Combined Terminal (ECT) in Rotterdam as an example, ECT utilizes both AGVs and lift AGVs to passively and actively handle the container transport AGVs, while the Hong Kong International Terminal (HIT) makes use of driveless transport vehicles, rail-mounted gantry cranes (RMGCs) and auxiliary systems. Some designs have shown performance improvement over that of the conventional terminals. However, a AGVs-based ACT requires intensive capital investment and high 
maintenance cost, which greatly reduces its attractiveness to port operators. Moreover, AGVs move slower than manned trailers and requires ancillary equipment (refueling and control) to serve the quay gantries. The shortcomings of AGVs in the existing ACT designs motivated the development of less expensive and more efficient new ACTs designs. Recently, Shanghai Zhenhua Port Machinery Company (ZPMC) introduced a new design of ACT which utilizes rail-mounted frame trolleys (FTs) and ground trolleys (GTs) to transport containers between the quay side and the yard side (Hu et al., 2013). Some quantitative contrastive analysis is proposed in the study by Zhen et al. and shows vast performance improvement over some conventional designs which are based on AGVs. Given this, the MLFB-based ACT handling system is proposed to achieve higher efficiency and lower cost.

The MLFB-based ACT handling system stems from AGV-based and FB-based ACT loading and unloading systems. In this system, the handling operations are implemented with the assistance of several handling machines, namely, MLFB, ALVs, FTs, RSs and elevators. The most innovative in this system is that the MLFB is applied to the yard instead of the original single-story rail way, which can enhance the efficiency of the whole system; In addition, the ALV is used for transportation of containers between the quayside and the yardside and it acts more as a connection function. For the evaluation process, a queuing theory model is used for analyzing the transport efficiency within the yard.

The remainder of this paper is organized as follows. Section 2 is the literature review which goes through the existing efforts in the conventional terminals or the ACTs. The proposed MFB-ACT handling system is discussed in Section 3 and the brief discussion of the functions of each subsystems are provided. Section 4 shows how this mode is established and conducts performance analysis on the efficiency of the new handling system. Section 5 summarizes the conclusions and practical implications.

\section{Literature Review}

Existing efforts have been devoted to the conventional terminals or the ACTs which are composed of AGV-based terminals, FB-based terminals and some other ACTs. With regard to the conventional terminals, Carlo et al. (2013) presented an in-depth overview of transport operations and the material handling equipment used. In this paper, current industry trends and developments were highlighted, and thus a new classification scheme for transport operations was proposed. $\mathrm{Ng}$ et al. (2005) proposed a branch and bound algorithm for the yard crane problem, which is subjected to a given set of loading and unloading jobs. Li et al. (2009) studied the yard crane scheduling problem and developed a mathematical model considering crane interference, fixed yard crane separation distances and simultaneous container storage/retrievals. Seungmo Kang et al. (2009) presented the mathematical models to optimize the size of transportation fleet (cranes and trucks) for unloading operations at container terminals. Zhen et al. (2011) proposed a mixed-integer programming model to integrate the berth template and the yard template planning. Chen et al. (2013) studied the interactions between crane handling and truck transportation in a maritime container terminal by addressing them simultaneously. Sharif et al. (2013) presented a novel approach for allocating containers to storage blocks in a marine container terminal. These researches were devoted to study the problem of schedule in container terminals without considerations of the simple utilization of automated equipment.

Recent advances in electronics, sensors, information technologies and automation have made the development of the ACTs feasible (Liu et al., 2002). Liu et al. (2004) used a multiple attribute decision making (MADM) method to assess the performance of the ACTs and determined the optimal number of deployed AGVs in each terminal. Referring to the AGV-based terminals, Tuan et al. (2006) presented a review on the design and 
control of the automated guided vehicle systems. Ghasemzadeh et al. (2009) presented an integrated algorithm for scheduling and routing AGVs in the mesh-like systems. Berman et al. (2009) studied a methodology for detailed evaluation of AGVs which included stand-alone sub-module evaluation, quantitative system evaluation and structured qualitative analyses. Hassan et al. (2011) defined a scheduling problem for AGVs in container terminals. Then this problem was solved by a novel algorithm, so called NSA+. Skinner et al. (2012) focused on scheduling for container transportation and encoded the problem using a two-part chromosome approach. Bocewicz et al. (2014) assumed a given topology of the MTN and schedules of operation sequences modeling concurrently manufactured product types. The main objective in that paper was to provide a declarative framework allowing one to adjust the AGVs fleet schedule.

In the recent years, a new design of ACT based on frame bridge was proposed by ZPMC. This handling system utilized rail-mounted frame trolleys (FTs) and ground trolleys (GTs) to transport containers in the quay and yard sides, respectively. With regard to the FB-based terminal, Zhen et al. (2012) made an explorative study to identify the challenges and opportunity for the FB-based terminals. Then quantitative comparisons were conducted to analyze the transport efficiency and stacking capacity between this new system and the widely used AGV-based system. Hu et al. (2014) studied a new ACT system which utilized multi-storey frame bridges for the link between the quayside and the yardside, which is different from the MLFB proposed in this study. Moreover, rail-mounted trolleys to transport containers between the quay and the yard. An iterative method was developed to analyze the operational efficiency of the ACT system.

Some other ACTs are existing in the world and many studies are illustrated as follows. Nguyen et al. (2009) discussed how to dispatch ALVs by utilizing information about pick-up and delivery locations and time in future delivery tasks. Then a mixed-integer programming model was provided for assigning optimal delivery tasks to ALVs. Cai et al. (2013) presented a multi-objective optimization model for the Autonomous Straddle Carriers Scheduling (ASCS) problem in ACTs.

As can be seen, there have been a wide range of researches for improving terminal operation efficiency. However, most of them concentrated on the logistic and/or operation strategies, no author has try to develop a concept of an integrated approach for the automation in the ACTs to meet the future demand. In this paper, the MLFB-based ACT handling system will be designed and its performance will be evaluated at the same time. In this system, the handling operations will be implemented with the assistance of four types of handling machines, namely, ALVs, elevators, FTs and RSs. For the evaluation process, a queuing theory model will be used for analyzing the transport efficiency within the yard.

\section{MLFB-based ACT Handling System}

\subsection{Configuration of the MLFB-based ACT Handling System}

As it is mentioned before, the MLFB-based ACT handling system takes advantages of several handling machines, such as MLFB, ALVs, elevators, FTs, RSs, double 40ft container quay cranes (QCs), rail-mounted gantry cranes(RMGs), external field trucks and so on. In this system, the most innovative is that the MLFB is applied to the yard instead of the original single-story rail way, which can enhance the efficiency of the whole system; Meanwhile, the ALV plays a role in the connection between the quay and the yard. Figure 1 is an illustration (not to scale) of the new ACT handling system. 


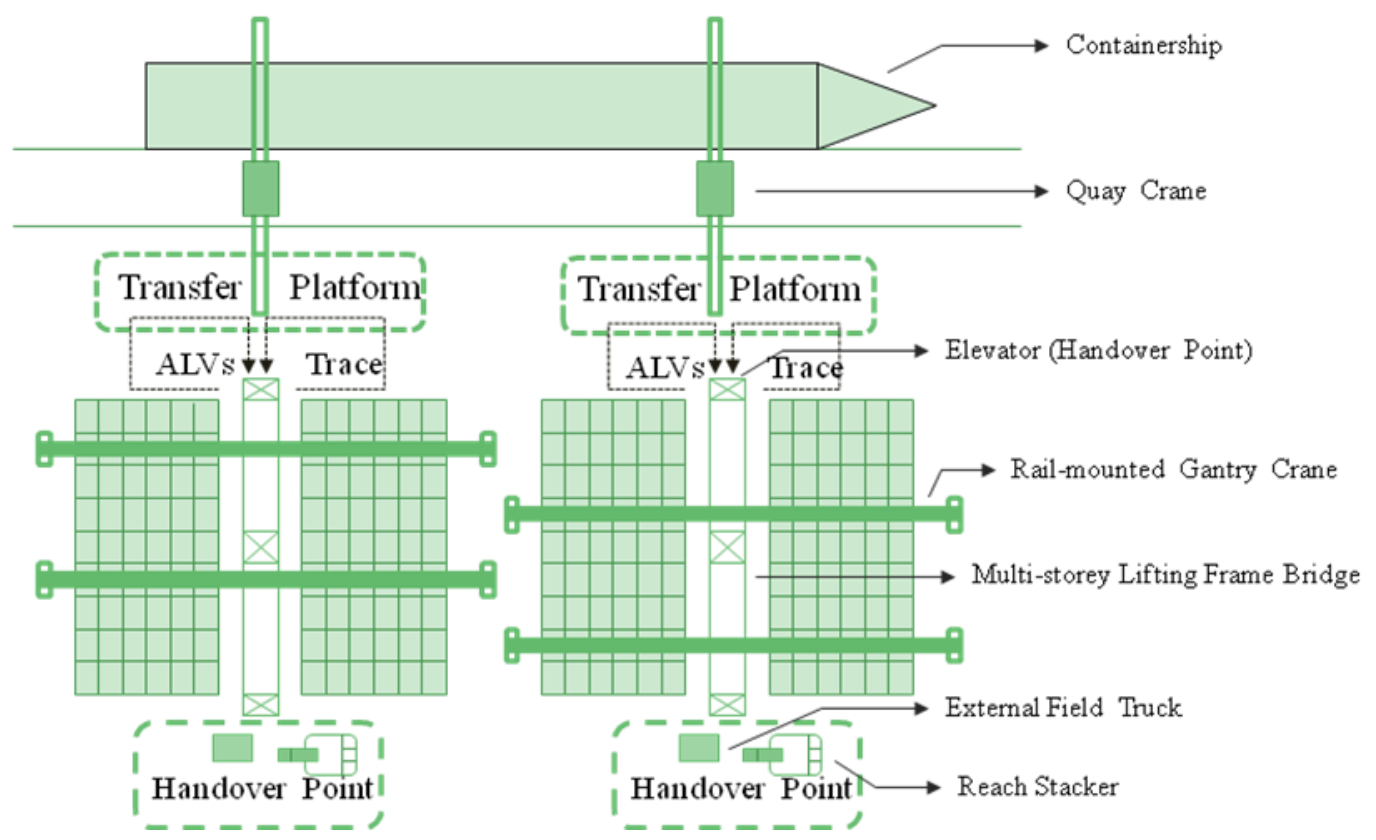

Figure 1. The Configuration of the MLFB-based ACT Handling System

As shown in Figure 1, ALVs travel between the quay side and handover points (HPs), and FTs travel in the area of handover points (HPs) and the yard side in the terminal. Obviously, In the MLFB system, HPs are the elevators that are utilized as the transfer platforms to transport the containers. In addition, several RSs are located at the rear of the MLFB. RSs replace RMGs and act as the communication between the yard and the external field. In this way, the RMGs do not need to move forth and back frequently, so that the energy consumption is reduced and the operating efficiency is increased. Moreover, the rear elevators serve as the handover points for external field trucks to collect and extract containers.

\subsection{Novel Integration of Equipment in the MLFB-based ACT Handling System}

From the above description, the new ACT handling system takes advantages of several handling machines, such as the MLFB, ALVs, elevators, FTs, double 40ft container quay cranes(QCs), rail-mounted gantry cranes(RMGs), external field trucks, RSs and so on. In this new system, these equipment is combined with each other and cooperates efficiently. Such novel integration of equipment issues a shining light as a handling system in the MLFB-based ACT, which may increase the performance of the terminal.

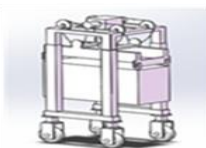

(a) ALV

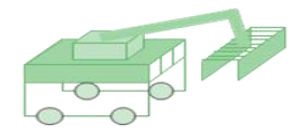

(b) R.S

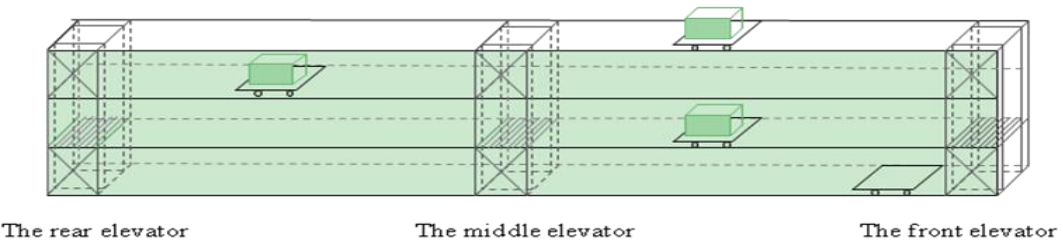

(c) The MLFB system

Figure 2. The Novel Integration of Equipment in MLFB-based ACT Handling System 
The ALVs are rarely used in practice unlike AGVs, which are applied widely in the existing ACTs in Rotterdam, Hamburger Harbour in the world. As described in the Figure 2(a), the ALVs are able to pick up and drop off containers without the assistance from other stacking equipment. In this way, it may reduce the waiting time caused by traffic congestion.

The RS is another spark in the combination. As illustrated in Figure 2(b), containers are transmit between the back elevator and external field truck by the RS rather than by the RMG, which reduces surplus moves of the RMG. Compared with the RMG, the RS is small and exquisite. Meanwhile, it is convenient, energy saving and pro-environment by using the RS.

The MLFB is the most innovative point in this ACT handling system. The biggest bright spot it owned is that the lifting up and down function can be implemented in the frame bridge. In this process, three kinds of elevators are designed to make the whole system active and efficient. As shown in the Figure 2(c), the front and rear elevators are used as the handover point. Several handling operations are designed in these elevators. The front elevator plays a role in connecting the quayside and the yardside, then the rear elevator acts as a tie between the yardside and the outfield; The middle elevator of the multistory frame bridge plays a communication role between the different layers.

The remain facilities are double 40ft container quay cranes (QCs), FTs, external field trucks and so on. They also play enormous roles in the loading and unloading operations.

\subsection{Technical Scheme of the Proposed ACT Handling System}

3.3.1. Handling Operation between the Quayside and the Yardside: As the world container shipping industry walks into the 10,000TEU mega ship era, the demand of improving the efficiency in the handling process is higher and higher. Due to this trend, it is necessary for terminal operators to design new kind of handling machines for the terminal which is capable to improve the efficiency of loading and unloading operations. Some measures are proposed to fulfill this requirement, e.g., increasing the number of QCs on each berth, using modern QCs which own higher performance, setting up multiple transfer platforms on the quayside.

Double 40ft container QCs are adopted in the quayside in the proposed ACT handling system. These settings are first developed by ZPMC. Meanwhile, multiple transfer platforms are set up under every quay crane in order to decrease the waiting time of each quay crane. By doing so, ALVs directly pick up containers without waiting, which tremendously increase the efficiency of the loading and unloading operations.

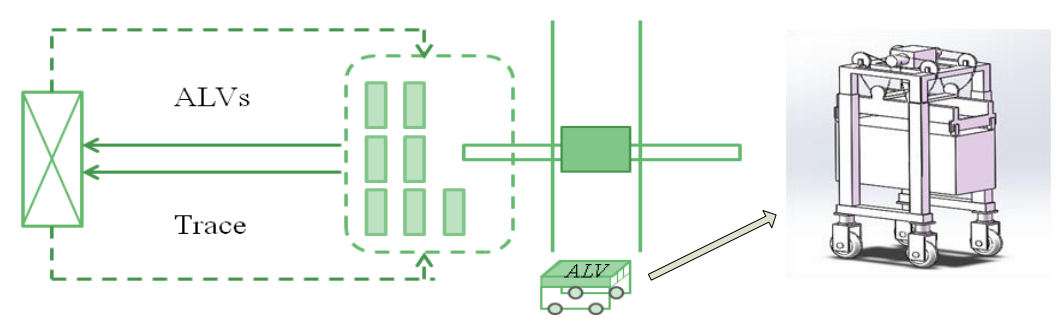

Figure 3. The Diagram of Handling Operation by ALVs between the Quayside and the Yardside

How ALV loads and unloads a container is shown in the Figure 3. As ALV is able to complete automated jobs, it saves labor involvement. Furthermore, ALV is charged via battery with diesel generator sets, which is energy economic. With the help of ALVs, QCs can directly load or discharge containers on the ground and do not need to wait for the prime moves. In this approach, ALVs can load one container and pass over another 
container on the ground. The scheduling of ALVs is supervised by the central control room in the terminal. This process can reduce the waiting time caused by traffic congestion and unbalanced operation capacity, and eventually improve the operational efficiency.

As illustrated in the Figure 3, ALVs are responsible for the transportation between the quayside and the yardside. The major advantage of the utilizing ALV is that it can pick up and drop off containers without the assistance from other stacking equipments. This eliminates the handshakes in the container handling process. Due to one elevator (handover point) is composed of two blocks, two ALVs cycles can work synchronously. In Figure 2, the double 40ft container quay crane put the containers into the transit platform and then ALV extracted the containers and moved them to the elevator along the middle route. After the handling operation is completed, ALV turns left or right to come back for the next handling operation. The route of ALVs can be described as two independent cycles.

3.3.2. Horizontal Transportation of Containers in the MLFB: In this system, ALVs and MLFB system are cooperatively applied to accomplish the horizontal transportation between the quay side and the yard side. ALVs are used for transmitting containers from the transfer platform in the quayside to the elevator in the yardside, and FTs on the MLFB are utilized to transport containers to the appointed container storage area.

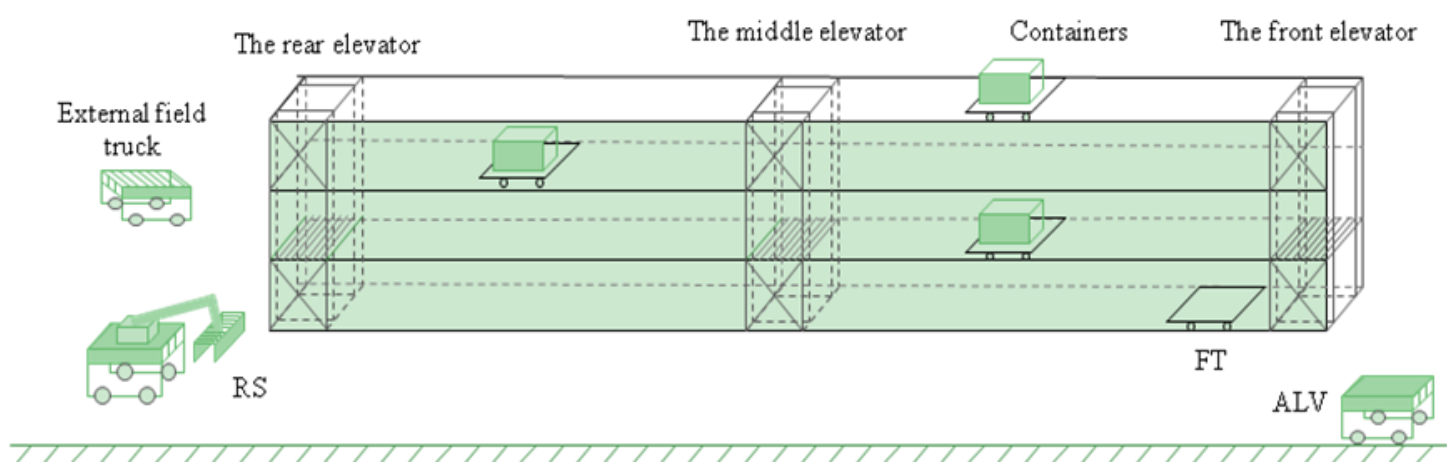

Figure 4. The Illustration of the MLFB and Handling Equipment

As shown in Figure 4, the elevators are located respectively at the front, middle and back of the lifting frame bridge system. The front elevator is used as a handover point which waits for containers extracted by ALVs and transports them to the specified layer (it does not need to move if containers are appointed to the first layer); The middle elevator of the MLFB plays a communication role between the different layers. It provides a chance for the flat cars to choose alternative way when the flat cars have been blocked in the rail. These operations can greatly enhance the flexibility of the handling system. In other word, the handling efficiency of the proposed ACT handling system is improved accordingly. The rear elevator is applied to transport containers to the external field truck, which utilizes FTs to transfer containers to the external field truck instead of RMGs, so that RMGs do not need to move back and forth, which enormously improve the efficiency of the terminal.

The FTs, elevators and frame bridges work together to implement the container loading and unloading operations. For the discharging process, the QCs can just drop the containers in the buffer area and go on to the next job. Then the ALVs pick up the containers, transport them to the appointed block, and drop them at the elevators in the front of each frame bridge. The containers will be transported by the flat cars to the assigned storage area, which will be picked up by the RMGs to the storage area. The loading process is just the reverse process of discharging. 
3.3.3. Transportation of Containers between the Yard Side and the External Field: According to the above procedure, the elevator in the rear of frame bridge is applied to transport containers to the external field truck with the help of handling mobile crane. In the import and export practices, loading and unloading containers is very important. So in this new design, elevators, FTs and RSs work orderly to deal with the collecting and extracting containers between the yardside and the external field. As shown in Figure 5, if containers are needed, the RMG will pick up the containers and then put them down onto the FT. Then the FT will move quickly to the elevator at the end of the frame bridge. Meanwhile, the RS will make some preparation to pick up the container to the external field truck. The collection of containers is the reverse process.

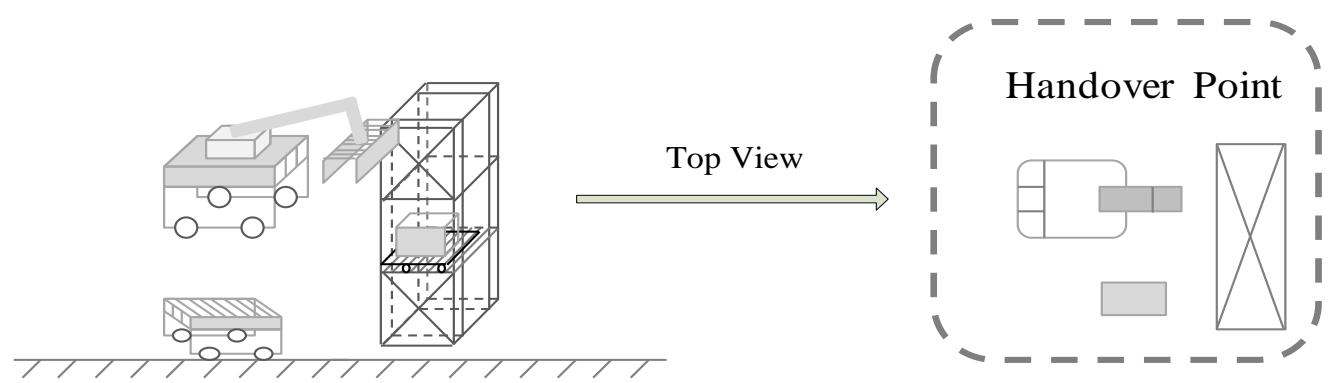

Figure 5. Transportation of Containers between the Yardside and the Outfield

3.3.4. Handling Process of the Proposed ACT Handling System: From the above description, the main equipments of the new handling system consists of double 40ft container quay cranes, ALVs, FTs, RMGs, RSs, and external field trucks. Multiple frame bridges decrease the waiting time of the yard crane. Moreover, the ALVs are used for horizontal transportation that eliminates the handshakes in the container handling process. In another meaning, the handling time of the whole system is reduced. The handling process of the MLFB-based ACT handling system is shown in the Figure 6.

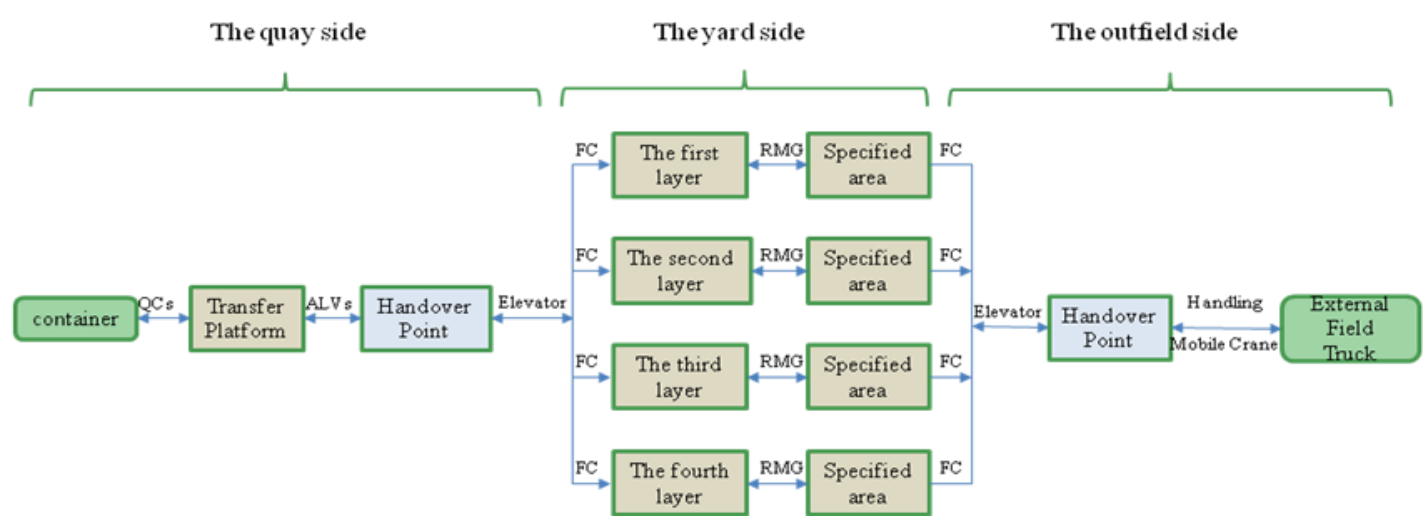

\section{Figure 6. The Handling Process of the MLFB-based ACT Handling System}

From Figure 6, it is concluded that the combination work of different handling facilities to transfer a container is the key for the high efficiency. The example of unloading process is illustrated as follows.

In the quayside, the ALV independently picks up a container and then moves towards the front elevator (handover point). When it arrives at the front elevator, the ALV put the container down on the FT, which is located at the elevator on the first layer. The ALV comes back for another practice and the FT is lifted by the front elevator to the appointed 
layer at the same time. Then the container is transported by the FT to the specified storage area for discharging activities. Meanwhile, the FT on the second layer moves onto the elevator which quickly falls onto the first layer in order to serve for the next container. With the arrival of the second container, the ALV picks up the container on the FT which is located at the elevator on the first layer. The rest will be done in the same manner.

In the yard side, the connection with the outside is conducted by the front-handling mobile crane. When containers are got from the block, the RMG will pick up the container and then put it down onto the FT. The handling mobile crane will make some preparations to pick up the container to the external field truck in the meantime.

\subsection{Main Features of the MLFB-based ACT Handling System}

3.4.1. An Integrated Approach in the Terminal: As is described before, an integrated approach is applied in this MLFB-based ACT handling system. The ALVs, frame bridge and elevators work collaboratively to pursue pro-environment, high efficiency and simple control. The ALVs can pick up and retrieve the containers by itself, so that it saves much idle time. In addition, the FTs on the frame bridge have a higher speed than the AGVs in the AGV-ACT, which can increase the transportation efficiency. The most important is that the elevators in the frame bridge perform perfect and act as different roles in the handling process. Due to the merits of each facility and the integration of the equipment, the possibility of the realization of high-efficient ACT handling system may be increased.

3.4.2. Multiple Transfer Platforms for the Quay Cranes in the Quayside: The throughput of the quay cranes has a major impact on the turnaround time of the vessels, which in turn affects the BOA. The double 40ft container quay crane can handle 35 containers per hour, but the actual performance depends on whether the quay crane can work with less waiting. Due to each quay crane has multiple access points and the ALV can pick up and drop off containers without the help from other stacking equipment so that quay cranes can work with less waiting time.

3.4.3. ALV for the Horizontal Transportation: The ALV can pick up and drop off containers without the help from other stacking equipment. Then the QCs can directly load or discharge containers on the ground and do not need to wait for the prime moves. In this new design, ALV can load one container and pass over another container on the ground. They are driven by electric to save energy and labor costs. ALV with hybrid energy can charge the battery with diesel generator sets. The scheduling of ALVs is controlled by the central control room in the terminal. It can reduce the waiting time caused by traffic congestion, unbalanced operation capacity and improve the operational efficiency.

3.4.4. Multi-storey Frame Bridge with Ability to Lifting Up and Down: In the MLFB-based ACT handling system, the MLFB with ability to lifting up and down can extend directly deep into the yard. There is a FT on each story of the MLFB and three elevators in the front, middle and back of the MLFB. With the FT transferring container to the dedicated storage, the RMG does not need to move forth and back to transport the container to the yard, which greatly reduces energy consumption. Elevators located at the front, middle and back of the frame bridge play different roles in the loading and unloading process respectively. The front elevator acts as a handover platform to establish the communication between ALVs and FTs; The middle elevator of the MLFB play a role in the communication between the different layers. It provides an chance for the FTs to chose another way When the FTs have blocked in the rail, which vastly enhances the flexibility of the handling system; The elevator locates at the rear of the MLFB is applied to transport containers to the external field truck, utilizing FTs instead of RMGs to 
transfer containers to the external field truck is saving energy and protecting the environment.

Compared with the handling system introduced by ZPMC, this MLFB system has advantages in transportation efficiency and flexibility. Due to the single-story frame bridge designed in ZPMC handling system, the rail-mounted trolleys need to wait for each other, which decreases the efficiency of the whole system. However, with the MLFB system used in the handling process, the transportation efficiency rises obviously because of the less waiting time of the trolleys.

3.4.5. Pickup and Retrieval of Containers with the Assistance of RSs: In this new design, several RSs act as transfer platforms transmitting containers between elevator and external field truck. Utilizing RSs instead of the RMGs to transfer containers to the external field truck is saving energy and protecting the environment. In addition, one RS is devoted to two blocks, which makes cost saved and source used.

In a word, the handling efficiency of the MLFB-based ACT handling system performs well with the cooperation of the ALVs, elevators, FTs and RSs. More or less, it may provide some new ideas for port designers in the future.

\section{Performance Analysis on the MLFB-based ACT Handling System}

Gross crane rate (GCR) is a key performance measure of a terminal (Liu et al., 2006). GCR is the total containers handled divided by the allocated crane time. In this study the yard side factor will be focused on and the transport efficiency within the yard will be considered based on the queuing theory.

Some frequently used parameters are shown in Table 1.

Table 1. The Notations of Some Parameters

\begin{tabular}{cl}
\hline Parameters & \multicolumn{1}{c}{ Specification } \\
\hline$h_{Y C}$ & the average handling and waiting time of YCs \\
$h_{Q C}$ & the average handling and waiting time of QCs \\
$t_{A L V}$ & expected travel time of ALVs \\
$t_{F T}$ & expected travel time of FTs \\
$v_{A L V}$ & velocity of ALVs \\
$v_{F T}$ & velocity of FTs \\
$w_{A L V}$ & expected waiting time of ALVs \\
$w_{F T}$ & expected waiting time of FTs \\
$\theta_{A L V}$ & expected cycle time of ALVs \\
$\theta_{Y C}$ & expected cycle time of FTs \\
$G, N$ & the number of ALVs or blocks \\
$B$ & the length of frame bridge \\
$\pi$ & average throughput rate of the new ACT \\
\hline
\end{tabular}

Several assumptions of this study are listed as follows.

(1) The pickup and delivery locations of these activities follow the uniform distribution along the quay in horizontal directions and along a side of a block in vertical directions, respectively. This assumption is commonly made in some analytical studies on container terminals (Kim et al., 2008).

(2) For the handling time of QCs and YCs, $\mathrm{h}_{\mathrm{QC}}$ is used to denote the average handling time of QCs and $h_{\mathrm{YC}}$ is used to denote the average handling times of YCs respectively in the new AVT handling system. In some literature on analysis of AGV-ACTs or 
conventional terminals (Lee et al., 2010), it is quite common to use the average handling times of QCs and YCs.

(3) To simplify the system the middle elevator of the frame bridge does not taken into consideration when the performance analysis is shown about the frame bridge. In addition, the handling operation between yardside and outfield using handling mobile crane does not taken into account. Only the transportation of containers between quayside and yardside is considered, then the performance of this handling process is analyzed.

(4) The front elevator acts as the handover point (HP), where is a place providing appropriate space to handing with numerous containers. In addition, the time of lifting up and down is ignored. Then only the travelling and waiting time of the FT are considered.

\subsection{Mathematical Analysis Model}

4.1.1 Model Description: First of all, the behaviors of handling equipments are analyzed. As shown in the Figure 7, there are two types of operation cycles related to ALVs and FTs, respectively for the loading and unloading activities. Those two types of cycles are linked up by the front elevator (the HP). From the view of the ALV, its cycle time consists of four elements in the unloading process, and the loading process still contains four stages similarly:

(1) the handling time of a $\mathrm{QC}\left(h_{Q C}\right)$ to load a container onto the transport platform;

(2) the travel time $\left(t_{A L V}\right)$ of the ALV from the pickup location in the quayside to the

HP in the yardside;

(3) the waiting time $\left(w_{A L V}\right)$ of a ALV at the HP and the handling time of the HP;

(4) the travel time $\left(t_{A L V}\right)$ of the ALV from the HP to the next pickup location.

Similarly, from the view of a FT, its cycle time also contains four stages for the discharging process and the loading process contains four stages as well:

(1) the waiting time of an FT $\left(w_{F T}\right)$ at a HP;

(2) the travel time $\left(t_{F T}\right)$ of the FT from the HP to the drop off position in the block;

(3) the waiting and handling time of a yard crane $\left(h_{Y C}\right)$;

(4) the travel time $\left(t_{F T}\right)$ of the other FT to return back to a HP

As shown in the Figure 7, the dashed line indicates that the other idle FT will come to the HP when the FT on the HP carries container to the dedicated block. By doing so, the cycle time of the FT only contains a $t_{F T}$, a $w_{F T}$ and a $h_{Y C}$.

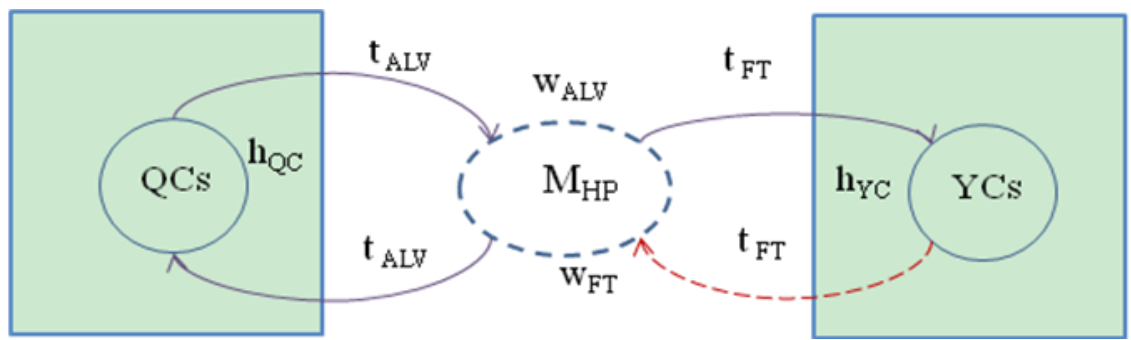

Figure 7. Model for the Single System

4.1.2. Mathematical Analysis: For the two types of cycles in the new handling system, $\theta_{A L V}$ is defined as the expected cycle time of the ALVs, and $\theta_{F T}$ is defined as the expected cycle time of the FTs.

$$
\begin{aligned}
& \theta_{A L V}=2 t_{A L V}+h_{Q C}+w_{A L V} \\
& \theta_{F C}=t_{F C}+h_{Y C}+w_{F T}
\end{aligned}
$$

From the handling process of the new system, the container is transported by the FT to the specified storage area for discharging activity, the FT on the other layer moves onto the elevator which quickly (as the dotted line in Figure 7) falls onto the first layer in order 
to serve for the next container at the same time. So the travel time of the FT to return back to a HP can be ignored for a discharging activity that the expected cycle time of FTs can be describe as the formula (2).

The new handling system has a large cycle, and there also exist some small cycles, which includes $M$ ALVs, $4 N$ FTs and $N$ YCs. Every loading or unloading job will go through the ALV cycle and one of the FT cycles. From the view of a long run, the average throughput by the $M$ ALVs and the $3 N$ FTs(actually there are only three FTs full-load work on the rail and the rest one is idle for the transportation )should be equal, which keeps the conservation of (loading or unloading) containers transported by these two types of cycles. For the new automated container terminal handling system, given the numbers of blocks $(N)$, ALVs $(M)$, the expected cycle time of ALVs $\left(\theta_{A L V}\right)$, and the expected cycle time of FTs $\left(\theta_{F T}\right)$, then we have

$$
3 N \times \theta_{A L V}=M \times \theta_{F C}
$$

$1 / \theta_{A L V}$ and $1 / \theta_{F C}$ reflect the average throughputs rates for one ALV and one FT, respectively. Hence, it can be concluded that

$$
\frac{3 N}{\theta_{F C}}=\frac{M}{\theta_{A L V}}
$$

Then the average throughput rate $\pi$ can be described as

$$
\pi=\frac{3 N}{\theta_{F C}}=\frac{M}{\theta_{A L V}}
$$

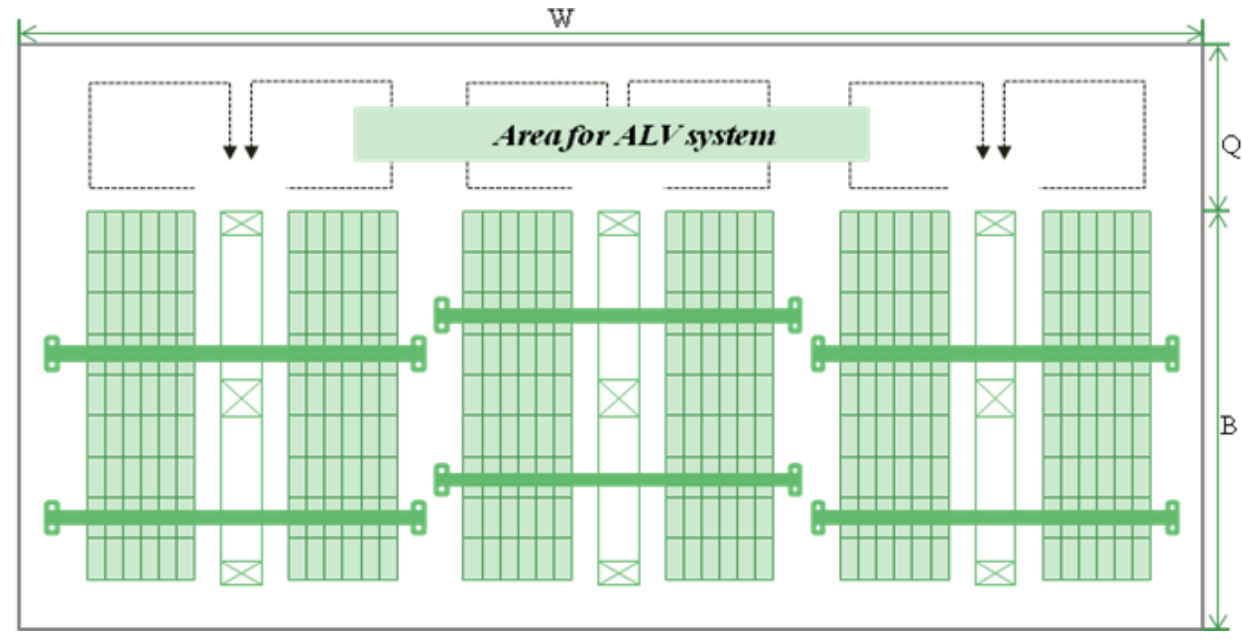

Figure 8. The Sketch of the Proposed ACT System (Not to Scale)

For getting the average throughput rate of the MLFB-based ACT handling system, it is necessary to calculate the expected values of $t_{F C}, t_{A L V}, w_{F C}$ and $w_{A L V}$.

\section{(1) The Expected Travel Time of FTs $\left(t_{F C}\right)$}

According to the assumption that the pickup and delivery locations of containers follow the uniform distribution along the wharf in horizontal directions and also along the blocks in vertical directions. So this activity follows uniform distributions $U(0, B)$ for the travel distance of FTs $\left(d_{F T}\right)$. B is the length of frame bridge (as shown in the Figure 8), then, it is can be got

$$
d_{F C}=\int_{0}^{B} x f(x) d x=B / 2
$$


Here B is the vertical length of the block. Given the velocity of FTs, the expected value of the travel time of FTs is calculated as follows

$$
t_{F C}=B / 2 v_{F C}
$$

Then the expected cycle time of FTs can be calculated as the formula (2).

\section{(2) The Expected Travel Time of ALVs $\left(t_{A L V}\right)$}

The analysis on the expected travel time of ALVs $\left(t_{A L V}\right)$ is similar as the analysis on the frame trolley in the FB-ACT proposed by ZPMC. However, the major difference lies in: $t_{F T}$ only considers the travel time in the horizontal direction; whereas the travel time in the vertical direction should also be added into $t_{A L V}$. So $t_{A L V}$ can be calculated by adding the vertical travel time, and Q is the vertical length of ALVs rails area (Zhen et al., 2012), the reasoning process is shown below:

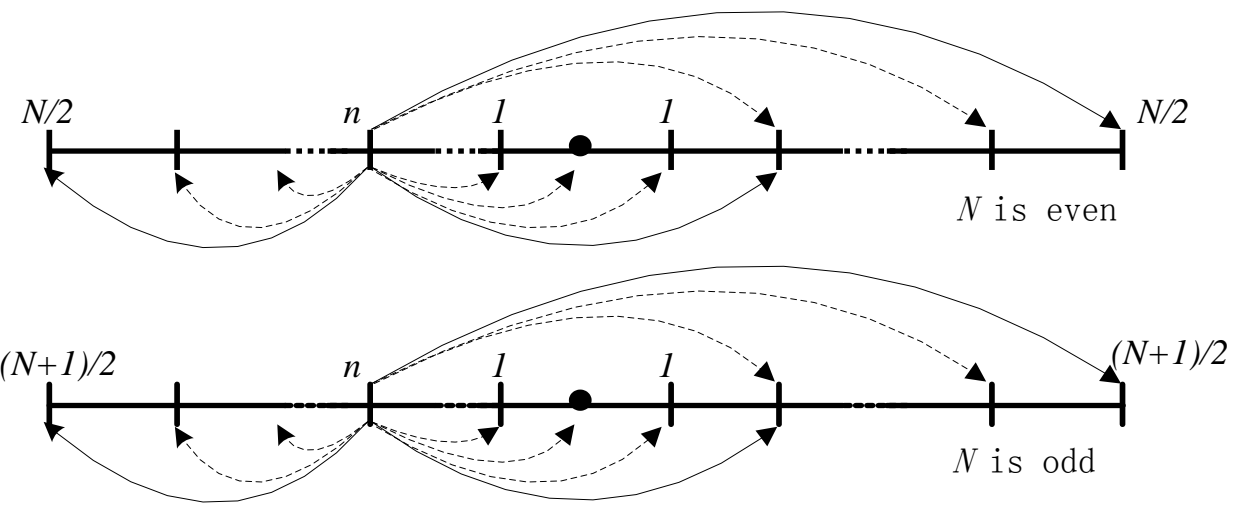

Figure 9. The Calculating of $d_{A L V}$

As shown in Figure 9, the berth is partitioned into segments; $N$ here is the number of blocks, and the blocks are named by index from the middle point (the black dot in Figure 9) to outside. It is assumed that all the pickups and deliveries occur at the center of the segment. The horizontal direction $\left(d_{A L V}^{H}\right)$ of the ALV is calculated as followed:

$$
\begin{aligned}
& \left\{\begin{array}{l}
d_{A L V}^{H}(n)=2 \sum_{i=1}^{\frac{N}{2}-n}\left(i \times \frac{W}{N}\right) \frac{1}{N}+\sum_{i=1}^{2 n-1}\left[\left(i+\frac{N}{2}-n\right) \frac{W}{N}\right] \frac{1}{N} \quad n \text { is even } \\
d_{A L V}^{H}(n)=2 \sum_{i=1}^{\frac{N+1}{2}-n}\left(i \times \frac{W}{N}\right) \frac{1}{N}+\sum_{i=1}^{2 n-2}\left[\left(i+\frac{N+1}{2}-n\right) \frac{W}{N}\right] \frac{1}{N} \quad n \text { is odd }
\end{array}\right. \\
& \left\{\begin{array}{l}
d_{A L V}^{H}(n)=\frac{W}{4 N^{2}}\left(N^{2}+4 n^{2}-4 n\right) \quad n \text { is even } \\
d_{A L V}^{H}(n)=\frac{W}{4 N^{2}}\left(N^{2}+4 n^{2}-8 n+3\right) \quad n \text { is odd }
\end{array}\right. \\
& \left\{\begin{array}{l}
d_{A L V}^{H}=2 \sum_{n=1}^{N / 2} d_{A L V}(n) \frac{1}{N} \quad n \text { is even } \\
d_{A L V}^{H}=d_{A L V}(1) \frac{1}{N}+2 \sum_{n=2}^{(N+1) / 2} d_{A L V}(n) \frac{1}{N} \quad n \text { is odd }
\end{array}\right.
\end{aligned}
$$




$$
d_{A L V}^{H}=\frac{W}{4}\left(1+\frac{N^{2}-4}{3 N^{2}}\right)
$$

The formula (8) illustrates the horizontal travelling direction of the ALV when $n$ is even and odd respectively, then the formula (8) can be simplified into formula (9). Since all the handling jobs are assumed to be equally distributed among all blocks, the horizontal travelling direction of the ALV can be obtained from the formula (11). Then the vertical distance is $Q$, so $t_{A L V}$ can be calculated as the formula (12):

$$
t_{A L V}=\frac{W}{4 v_{A L V}}\left(1+\frac{N^{2}-4}{3 N^{2}}\right)+\frac{Q}{v_{A L V}}
$$

\section{(3) The Expected Waiting Time of ALVs $\left(w_{A L V}\right)$}

The method of queuing theory is employed to estimate the waiting time of ALVs. The ALVs can be regarded as "customers" and the FTs in each frame bridge can be seen as the parallel "servers." In the paper, this process is modeled as the $M / M / s$ queuing system, here $\mathrm{s}$ is the number of FTs, $\mathrm{s}=4$. According to the formula of the mean waiting time for the $M / M / s$ queuing system, the waiting time of ALVs is calculated as followed:

$$
w_{A L V}=\frac{(s \rho)^{s}}{s !} \cdot\left[(1-\rho) \sum_{n=0}^{s-1} \frac{(s \rho)^{n}}{n !}+\frac{(s \rho)^{s}}{s !}\right]^{-1} \cdot \frac{T}{(1-\rho) \cdot s}
$$

In the equation (9), $\mathrm{T}$ is on behalf of the expected handling time of "servers," such as the FTs; $\rho$ is the traffic intensity which is based on the average arrival rate $(\lambda)$ of the "customers," such as the ALVs. Suppose for a long period $\tau$ the number of cycles for one ALV is $\tau / \theta_{A L V}$; the total number of cycles for all the $M$ ALVs is $M \cdot \tau / \theta_{A L V}$. So there are $M \cdot \tau /\left(\theta_{A L V} \cdot N\right)$ cycles allocated to each FT during the period $\tau$. Hence, the average arrival rate of ALVs is $M /\left(\theta_{A L V} \cdot N\right)$ (Zhen al., 2012). Then $\rho$ can be got by the following equations:

$$
\begin{aligned}
& T=t_{F C}+h_{Y C}=\frac{B}{2 v_{F C}}+h_{Y C} \\
& \rho=\frac{\lambda \cdot T}{s}=\frac{M \cdot\left(\frac{B}{2 v_{F C}}+h_{Y C}\right)}{\mathrm{s} \cdot N \cdot\left(2 \mathrm{t}_{\mathrm{ALV}}+\mathrm{h}_{\mathrm{QC}}+\mathrm{w}_{\mathrm{ALV}}\right)}
\end{aligned}
$$

From the above equation (9) and equation (11), $w_{A L V}$ can be obtained. In reality, the four "servers" (FTs) serve for the ALVs all the time, so the waiting time of the ALVs can be ignored in the long run. In that case, $w_{A L V}$ is not considered in the next work.

\section{(4) The Expected Waiting Time of FTs $\left(w_{F C}\right)$}

From the equation (1), (2) and (4), $w_{F C}$ can be obtained:

$$
\mathrm{w}_{\mathrm{FC}}=\frac{3 N}{M} \cdot\left(2 t_{A L V}+h_{Q C}\right)-t_{F C}-h_{Y C}
$$

\section{(5) The Average Throughput Rate of the New Designed ACT}

According to the analysis on the travel time and waiting time of the ALVs or FTs, the average throughput rate of the new designed ACT is represented as followed: 


$$
\pi=\frac{3 N}{\theta_{F C}}=\frac{M}{\theta_{A L V}}=\frac{M}{2\left(\frac{W}{4 v_{A L V}}\left(1+\frac{N^{2}-4}{3 N^{2}}\right)+\frac{Q}{v_{A L V}}\right)+h_{Q C}}
$$

\subsection{Results Analysis}

In order to analyze the performance of the new handling system, some different cases are put forwards hereinafter. Define $N=10, M=10$, then the new handling system can be divided into 5 small systems and each system has 2 ALVs, 4 FTs. Then the impact of different size of blocks is discussed in table.2. As shown in the table.2, the width of the terminal changes from $400 \mathrm{~m}$ to $1200 \mathrm{~m}$, the velocity of FTs is described as $v_{F C}=300$ $\mathrm{m} / \mathrm{min}$ and the velocity of ALVs is shown as $v_{A L V}=230 \mathrm{~m} / \mathrm{min}$. In addition, the handling time of $\mathrm{YC}$ is $h_{Y C}=3 \mathrm{~min} /$ container and the handling time of QC is $h_{Q C}=2 \mathrm{~min} /$ container. In the table.3, the different velocities of ALVs (changing from $200 \mathrm{~m} / \mathrm{min}$ to $300 \mathrm{~m} / \mathrm{min}$ ) are analyzed and the change of the average throughput rate of the new handling system are obtained. Obviously, with the increasing of $v_{A L V}$ the average throughput rate is increased; The different handling time of QCs (changing from $1.0 \mathrm{~min} /$ containers to $3.5 \mathrm{~min} /$ containers) are shown in the Table 4; And Table 5 illustrates how the change of $Q$ influences the average throughput rate.

The results are demonstrated in the following:

(1) With the increase of horizontal length of the port, the average running distance for ALVs is increased, then the average throughput rate $\pi$ for single system is decreased accordingly from Table 2. However, the average throughput rate of the whole terminal is increased due to the increasing blocks. In addition, the storage capacity of blocks is improved as the increasing of horizontal length of the terminal, which may enhance the performance of the terminal.

(2) As shown in Table 3, the average throughput rate is improved with the rising velocities of ALVs. So it is necessary to study and design faster ALVs, however, it may be hindered by the current technology. Even if the hamper of the technology, it is confident of designers to develop more efficient trolleys.

(3) The Table 4 illustrates how the handling time of QCs to influence the average throughput rate of the proposed ACT system. When the handling time of QCs reduces, the average throughput rate of the handling system increases obviously. In other word, the quay crane may be the bottleneck of new the handling system. This will provide terminal operators some reference in the practice operations.

(4) Table 5 shows how the change of $Q$ influences the average throughput rate. In the following table, the $Q$ is changed from $20 \mathrm{~m}$ to $60 \mathrm{~m}$, and the results are changed slightly. This result may be caused by the multiple transfer platforms in the quay side that the ALV does not need to wait for QCs.

Table 2. The Results for Different Size of the Blocks

\begin{tabular}{ccccc}
\hline$W(m)$ & $N$ & $v_{A L V}(\mathrm{~m} / \mathrm{min})$ & $h_{Q C}($ min/container $)$ & $\pi($ containers $/$ min $)$ \\
\hline 400 & 4 & 230 & 2 & 11.36 \\
600 & 6 & 230 & 2 & 14.54 \\
800 & 8 & 230 & 2 & 16.96 \\
1000 & 10 & 230 & 2 & 18.90 \\
1200 & 12 & 230 & 2 & 20.38 \\
\hline
\end{tabular}


Table 3. The Results for Different Velocities of ALVs

\begin{tabular}{ccccc}
\hline$W(m)$ & $N$ & $v_{A L V}(\mathrm{~m} / \mathrm{min})$ & $h_{Q C}($ min/container $)$ & $\pi($ containers/min $)$ \\
\hline 1000 & 10 & 200 & 2 & 17.2 \\
1000 & 10 & 220 & 2 & 18.3 \\
1000 & 10 & 240 & 2 & 19.4 \\
1000 & 10 & 260 & 2 & 20.3 \\
1000 & 10 & 280 & 2 & 21.2 \\
1000 & 10 & 300 & 2 & 22.1 \\
\hline
\end{tabular}

Table 4. The Results for Different Handling Time of QCs

\begin{tabular}{ccccc}
\hline$W(m)$ & $N$ & $v_{A L V}(\mathrm{~m} / \min )$ & $h_{Q C}($ min/container $)$ & $\pi_{(\text {containers/min })}$ \\
\hline 1000 & 10 & 230 & 1.0 & 23.2 \\
1000 & 10 & 230 & 1.5 & 20.8 \\
1000 & 10 & 230 & 2.0 & 18.9 \\
1000 & 10 & 230 & 2.5 & 17.2 \\
1000 & 10 & 230 & 3.0 & 15.9 \\
1000 & 10 & 230 & 3.5 & 14.7 \\
\hline
\end{tabular}

Table 5. The Results for Different Length of the ALVs Vertical Rail

\begin{tabular}{cccccc}
\hline$W(m)$ & $N$ & $v_{A L V}(\mathrm{~m} / \mathrm{min})$ & $h_{Q C}(\mathrm{~min} /$ container $)$ & $Q(m)$ & $\pi($ containers/min $)$ \\
\hline 1000 & 10 & 230 & 2 & 20 & 19.8 \\
1000 & 10 & 230 & 2 & 30 & 19.5 \\
1000 & 10 & 230 & 2 & 40 & 19.1 \\
1000 & 10 & 230 & 2 & 50 & 18.9 \\
1000 & 10 & 230 & 2 & 60 & 18.5 \\
\hline
\end{tabular}

\section{Conclusions}

This paper introduces the MLFB-based ACT handling system which utilizes ALVs, MLFB and FTs to transport containers between the quay and the yard, and makes an explorative study based on the queuing theory to analyze the transport efficiency within the yard. An analytical model and performance measure are proposed for the MLFB-based ACT handling system. The major contributions of the study are:

(1) This study introduces a new type of ACT handing system, which can be named MLFB-based ACT handling system. It develops a concept of an integrated approach for the automation in the designed ACT system, and the integration of different automated equipment makes the whole handling system more pro-environment, high efficiency and simple control..

(2) In this handling system, the most innovative is the MLFB. It is applied to the transportation in the yard instead of the original single-story rail, which can enhance the efficiency of the whole system. There are three elevators in the front, middle and back of the MLFB, and they act as different roles to make the MLFB system flexible.

(3) In the quayside, multiple transfer platforms are designed for the QCs. The ALV is a link between the quay and the yard, and it can pick up containers without the assistance of other stacking equipment so that the QCs can work with less waiting time.

However, there are still some limitations for this study. For the interest of simplicity, only the performance of the front elevator is focused on and the middle and the rear elevators are not taken into consideration; This study is based on several assumptions, e.g., all the blocks are in operations simultaneously, all berth segments along the quay are in full load condition, the loading and unloading tasks are evenly distributed along the vertical (along blocks) and horizontal (along the quay) directions. Actually, these 
situations seldom exist in the realistic port environments. The derived measures (formula) may not reflect the realistic performances (Zhen et al., 2012).

In the future study, it may be necessary to take the performance of the middle and the rear elevators of the MLFB-based handling system into consideration. Different dispatching strategies among ALVs, elevators, and FTs are needed to be analyzed. Moreover, uncertain factors in the port operations in ACTs will be investigated in the future (Hu al., 2013).

\section{Acknowledgements}

This work is sponsored by National Natural Science Foundation project (71101090), Shanghai Top Academic Discipline Project-management science \& engineering, Shanghai Yangfan Program (14YF1411200), Shanghai Municipal Education Commission Project (13YZ080, 14YZ112), Doctoral Fund of the Ministry of Education (20133121110001) and Shanghai Maritime University Research Project (20120102). We also thank anonymous referees and the editor-in-chief.

\section{References}

[1] H. D. Le-Griffin and M. Murphy, "Container terminal productivity: experiences at the ports of Los Angeles and Long Beach," in National Urban Freight Conference, Long Beach, Calif., USA, (2006).

[2] H. U. Hongtao, B. K. Lee, Y. Huang, L. H. Lee and E. P. Chew, "Performance Analysis on Transfer Platforms in Frame Bridge Based Automated Container Terminals", Mathematical Problems In Engineering, (2013).

[3] H. J. Carlo, I. F. A. Vis and K. J. Roodbergen, "Transport Operations in Container Terminals: Literature Overview", Trends, Re-search Directions and Classification Scheme," European Journal of Operational Research, vol. 236, no. 1, (2014), pp. 1-13.

[4] W. C. Ng and K. L. Mak, "Yard crane scheduling in port container terminals" Applied Mathematical Modelling, vol. 29, (2005), pp. 263-276.

[5] W. Li , Y. Wu , M. E. H. Petering, M. Goh, and R. D. Souza, "Discrete time model and algorithms for container yard crane scheduling", European Journal of Operational Research, vol. 198, no. 1, (2009), pp. $165-172$.

[6] S. Kang, J. C. Medina and Y. Ouyang, "Optimal operations of transportation fleet for unloading activities at container ports", Transportation Research, vol. 42, (2008), pp. 970-984.

[7] L. Zhen, E. P. Chew, and L. H. Lee, "An integrated model for berth template and yard template planning in transshipment hubs", Transportation Science, vol. 45, no. 4, (2011), pp. 483-504.

[8] L. Chen, A. Langevin and Z. Lu, "Integrated scheduling of crane handling and truck transportation in a maritime container terminal”, European Journal of Operational Research, vol. 225, no. 1, (2013), pp. $142-152$.

[9] O. Sharif and N. Huynh, "Storage space allocation at marine container terminals using ant-based control”, Expert Systems with Applications, vol. 40, (2013), pp. 2323-2330.

[10] C.-I. Liu, H. Jula, K. Vukadinovic and P. Ioannou, "Automated guided vehicle system for two container yard layouts", Transportation Research, vol. 12, (2004), pp. 349-368.

[11] C.-I. Liu, H. Jula and P. A. Ioannou, "Design, Simulation, and Evaluation of Automated Container Terminals", Transactions on intelligent transportation systems, vol. 3, no. 1, (2002).

[12] L.-A. Tuan and M. B. M. De Koster, "A review of design and control of automated guided vehicle systems", European Journal of Operational Research, vol. 171, (2006), pp. 1-23.

[13] H. Ghasemzadeh, E. Behrangi and M. A. Azgomi, "Conflict free scheduling and routing of automated guided vehicles in mesh topologies", Robotics and Autonomous Systems, vol. 57, (2009), pp. 738-748.

[14] S. Berman, E. Schechtman and Y. Edan, "Evaluation of automatic guided vehicle systems", Robotics and Computer-Integrated Manufacturing, vol. 25, (2009), pp. 522-528.

[15] H. Rashidi and E. P. K. Tsang, "A complete and an incomplete algorithm for automated guided vehicle scheduling in container terminals", Computers and Mathematics with Applications, vol. 61, (2011), pp. 630-641.

[16] G. Bocewicz, I. Nielsen and Z. Banaszak, "Automated guided vehicles fleet match-up scheduling with production flow constraints", Engineering Applications of Artificial Intelligence, vol. 30, (2014), pp. 49-62.

[17] B. Skinner, S. Yuan, S. Huang, D. Liu, B. Cai, G. Dissanayake, H. Lau, A. Bott and D. Pagac, "Optimisation for job scheduling at automated container terminals using genetic algorithm", Computers \& Industrial Engineering, vol. 64, (2013), pp. 511-523. 
[18] H. Hu, Y. Huang, L. Zhen, B. K. Lee, L. H. Lee and E. P. Chew, "A decomposition method to analyze the performance of frame bridge based automated container terminal", Expert Systems with Applications, vol. 41, no. 2, (2014), pp. 357-365.

[19] L. Zhen, L. H. Lee, E. P. Chew, D.-F. Chang and Z. X. Xu, "A Comparative Study on Two Types of Automated Container Terminal Systems", IEEE Transactions on Automation Science and Engineering, vol. 9, no. 1, (2012), pp. 56-69.

[20] V. D. Nguyen and K. H. Kim, "A dispatching method for automated lifting vehicles in automated port container terminals", Computers \& Industrial Engineering, vol. 56, (2009), pp. 1002-1020.

[21] B. Cai, S. Huang, D. Liu, S. Yuan, G. Dissanayake, H. Lau and D. Pagac, "Multi-objective Optimization for Autonomous Straddle Carrier Scheduling at Automated Container Terminals", IEEE Transactions on Automation Science and Engineering, vol. 10, no. 3, (2013), pp. 711-725.

[22] J. Y. Liu, Y. W. Wan, and L. Wang, "Quay crane scheduling at container terminals to minimize the maximum relative tardiness of vessel departures", Nav. Res. Logist. , vol. 53, (2006), pp. 60-74.

[23] K. H. Kim, Y. M. Park, M. J. Jin, “An optimal layout of container yard”, OR Spectrum, vol. 30, (2008), pp. 675-695.

[24] B. K. Lee and K. H. Kim, "Optimizing the block size in container yards", Transp. Res. Part E, vol. 46, (2010), pp. 120-135.

\section{Authors}

Ting Fang, he is a postgraduate in Shanghai Maritime University, China. Her main research area is about design and evaluates the handling system of automated container terminal.

Wei Yan, he received his PhD from Nanyang Technological University (NTU), Singapore. Currently, He is working as the director of Development Planning Division, SMU and the deputy director of Engineering Research Center for Container Supply Chain Technology, MOE, China. He has been entitled as the committee member of the Shanghai Shuguang Scholar, etc. He completed 1 state-key textbook, over 10 patents, and more than 100 publications, including over $30 \mathrm{SCI} / \mathrm{SSCI}$-indexed papers as well as more than 250 SCI citations.

Youfang Huang, he is a Professor, and President at Shanghai Maritime University, China. His research work is focused on logistics management and engineering. He has a BEng in Mechanical Engineering from Shanghai Maritime University, and a MEng and a $\mathrm{PhD}$ in Mechanical Engineering from Tongji University in Shanghai, China. He is also an international consultant of UNCTAD/WTO, the chairman of Logistics Association in Northeast Asia, and the deputy president of Chinese Logistics Association.

Junliang He, he is a lecturer in Shanghai Maritime University, China. He has a BEng in Industrial Engineering and a MEng in Mechanical Engineering from Shanghai Maritime University, China, and a PhD in Mechanical Manufacture and Automation from Tongji University, China. His main research areas are logistics engineering and operation research.

Danping Lin, he is a lecturer in Shanghai Maritime University, China. She has a bachelor degree in Information Management and Information System from Xiamen University, Xiamen, China, and a $\mathrm{PhD}$ in System and Engineering Management from Nanyang Technological University, Singapore. Her research domain includes supply chain management, production research and port management. 
International Journal of Hybrid Information Technology

Vol. 8, No. 4 (2015) 